\title{
The impact of mild closed head injury on involuntary saccadic adaptation: Evidence for the preservation of implicit motor learning
}

\author{
MARCUS H. HEITGER ${ }^{1,2}$, MICHAEL R. MACASKILL ${ }^{1,2}$, RICHARD D. JONES $^{1,2,3}$ \& \\ TIM J. ANDERSON ${ }^{1,2,4}$ \\ ${ }^{1}$ Christchurch Brain Research Group, Christchurch, New Zealand, ${ }^{2}$ Department of Medicine, Christchurch School of \\ Medicine $\mathcal{E}$ Health Sciences, Christchurch, New Zealand, ${ }^{3}$ Department of Medical Physics and Bioengineering, and \\ ${ }^{4}$ Department of Neurology, Christchurch Hospital, Christchurch, New Zealand
}

\begin{abstract}
Objective: Mild closed head injury (CHI) can impair performance on volitional saccades (fast eye movements), with poorer saccade accuracy being one of the principal deficits. Assessing a patient group with known deficits of volitional saccades, the authors investigated whether mild CHI similarly impairs the implicit adaptation of visually-guided (reflexive) saccades, an important process which maintains saccadic accuracy.

Methods: Within 2 weeks following mild CHI, 30 patients and 30 matched controls were compared on a computerized paradigm, which artificially induced saccadic adaptation. In response to an initial stimulus, subjects made a saccade during which the stimulus was displaced centripetally causing the initial (primary) saccade to be inaccurate. While these intra-saccadic changes remained unnoticed by the subjects, the displacements gradually caused adaptive saccadic hypometria.

Results: No differences in adaptation were found between the CHI group and the controls $(F(1,29)=0.51, p=0.48)$. This finding indicates that mild CHI does not impair implicit reflexive saccade adaptation and suggests that cerebellar function and functions of deeper brain structures such as the thalamus, superior colliculus and the basal ganglia may be largely preserved following mild CHI. The current results support the notion that the profile of oculomotor function after mild $\mathrm{CHI}$ reflects a centripetal gradient of impairment and relates closely to the functional integrity of the injured brain.
\end{abstract}

\section{Introduction}

Mild closed head injury (CHI) has been shown to cause considerable neural injury throughout the brain [1-7]. On the Glasgow Coma Scale (GCS), the most frequently used clinical tool to grade head injury severity, scores between 13 and the maximum of 15 are classified as mild cases. The traditional interpretation of the term 'mild' CHI includes a brief loss of consciousness in combination with a post-traumatic amnesia (PTA) duration of less than 24 hours followed by disturbances of neurological function [8]. Although mild $\mathrm{CHI}$ can produce focal lesions in fronto-temporal cerebral areas, most of the neural damage occurs in the form of non-focal diffuse axonal injury (DAI), which results from the rotational shear forces occurring in the brain at the time of the injury. The degree of DAI is related to head trauma severity and decreases centripetally from cortical to subcortical structures [9-11], although it can extend into the deeper white matter and into the brain stem [12].

The cerebral networks concerned with the control of saccades are of considerable complexity and incorporate several cortical and sub-cortical structures as well as the cerebellum. These networks are susceptible to the adverse effects of neural injury: mild CHI causes deficits of volitional saccades such as antisaccades, sequences of memory-guided saccades and self-paced saccades, while reflexive saccades remain unaffected $[13,14]$. The corresponding saccadic deficits reported previously by the authors comprised prolonged latencies of pro-saccadeerrors in the anti-saccade task, a trend to longer anti-saccade latencies and prolonged inter-saccadic latencies of self-paced saccades following mild CHI. In addition, the group with CHI showed an increased number of directional errors on sequences of memory-guided saccades, marginally increased numbers of directional errors in the anti-saccade task and markedly decreased accuracy of anti- and

Correspondence: Marcus Heitger, Van der Veer Institute for Parkinson's and Brain Research, 16 St Asaph Street, PO Box 2682, Christchurch 8001, New Zealand. E-mail: marcus.heitger@chmeds.ac.nz

(Received 4 August 2003; accepted 29 April 2004). 
memory-guided saccades, with larger absolute position errors and hypermetria of saccades in both these paradigms. The same patient group was also tested on the paradigm examining implicit (subconscious) adaptation of visually-guided (reflexive) saccades presented herein. This paradigm assessed motor functions, which, in contrast to volitional saccades, elude conscious control and are mediated predominantly by deeper, sub-cortical brain structures and the cerebellum. Hence, this experiment examined aspects of oculomotor function that are not otherwise accessible by volitional motor tasks and aimed to provide information on the impact of mild head trauma on implicit motor learning.

The adaptation of saccadic gain (eye movement amplitude relative to distance to target) based on the accuracy of prior saccades is an important mechanism of the brain to maintain the accuracy of saccades in a natural environment. This adaptation process is essential for saccadic accuracy as the short duration of saccades $(<100 \mathrm{~ms})$ prevents the 'in-flight' incorporation of visual feedback. Consequently, the amplitude of each saccade is programmed before its initiation and cannot be altered once the eye movement has been initiated. Most saccades are very close to the intended amplitude, yet processes such as ageing and disease may reduce their accuracy over time. In order to maintain saccade accuracy, systematic error signals are taken into account for the programming of future saccades of similar size and direction via the process of saccadic adaptation. Saccadic adaptation constitutes a form of implicit (subconscious) learning and the viewer remains unaware of it. This adaptation process enables the brain to compensate for the adverse effects of ageing or disease on oculomotor accuracy (see MacAskill et al. [15] for a review) and is observed in myasthenia gravis [16], oculomotor paresis [17], oculomotor muscle weakness [18] or macular degeneration [19].

Although saccadic adaptation occurs naturally, it can also be artificially induced in the laboratory using surreptitious intra-saccadic target displacements to experimentally introduce saccadic inaccuracy. The experimental principle to introduce saccadic adaptation was first put into practice by McLaughlin [20]. Since then, many studies have examined the phenomenon of saccadic adaptation [21-29] and the saccadic suppression of displacement, that is the inability of the viewer to perceive intra-saccadic target shifts [30].

Over recent years, it has become apparent that the involvement of cerebral regions in the mediation of saccadic adaptation varies between different saccadic tasks. Lesion studies [31-33] and imaging evidence [34] indicate that the cerebellum is predominantly responsible for the adaptation of visually-guided (reflexive) saccades. The adaptation of general volitional saccades, however, may be mainly mediated by the frontal eye field (scanning saccades) or the dorsolateral pre-frontal cortex (memory-guided saccades) [35]. In addition, there are suggestions that structures which are functionally further 'downstream' in the initiation of saccades, such as the basal ganglia, are also important for these adaptation processes [29].

This study aimed to measure the impact of mild $\mathrm{CHI}$ on reflexive saccade adaptation and is, to the authors' knowledge, the first to address implicit motor learning in the context of head trauma.

\section{Methods}

\section{Subjects}

Thirty patients (11 female and 19 male) with mild CHI (Glasgow Coma Scale score 13: two cases, 14: five cases, 15: 23 cases) were recruited from patients presenting with acute head injury to Christchurch Hospital (the principal hospital for a population pool $>400000$ within the South Island of New Zealand). Each of the 30 patients had experienced post-traumatic amnesia ranging between 3 minutes and 4 hours (mean $=34.4$ minutes). Twenty-five patients had a confirmed loss of consciousness (mean $=2.6$, range $1-15$ minutes). Mean age was 22.2 years (SD 7.1, range $15-37$ years) and mean years of education was 12.8 (SD 1.86). CT head scans were undertaken in seven $\mathrm{CHI}$ subjects and all were normal. All patients were either employed or attended institutions for secondary or tertiary education and none was involved in litigation. Exclusion criteria were: evidence of any influence of alcohol or psychoactive drugs at the time of injury, regular intake of psychoactive drugs or a history of drug abuse, central neurological disorder or psychiatric condition, structural brain damage or haematoma on CT head scan (were obtained), oculomotor or somatomotor deficits upon clinical examination, presence of strabismus, Snellen visual acuity worse than $6 / 12$, skull fractures or prior history of mild, moderate or severe head injury with persisting symptoms or complaints.

The control group consisted of subjects with no history of mild, moderate or severe head injury with persisting symptoms or complaints, no central neurological disorder or psychiatric condition and no regular intake of psychoactive drugs or history of drug abuse. The controls were matched with respect to age (within 3 years for patients $>18$ years, within 1 year for patients $<18$ years), gender and educational background (years of formal education, within 2 years for patients $>18$, within 1 year for subjects $<18)$. Mean age for the control group was 
22.4 years (SD 7.0, range 15-37 years) and mean years of education was 13.2 (SD 2.1). The project was approved by the local ethics committee and written consent was obtained from all participants. Subjects were compensated for their travel costs to attend the testing at the hospital but received no other payments.

\section{Apparatus and procedure}

All patients were tested within 16 days of their injury ( $M=6.46$, SD 3.3, range 3-16 days). Eye movements were recorded using the infra-red scleral reflection oculography technique [36] (IRIS, Skalar Medical, BV, Delft, The Netherlands). Eye position signals were low-pass filtered at $100 \mathrm{~Hz}$, sampled and digitized at $200 \mathrm{~Hz}$ and recorded by a PC for off-line analysis [37]. Subjects were seated in a darkened room. Head movements were stabilized via a wax bite-bar. Eye movements were elicited by instructing the subject to follow a computer-generated stimulus (a red square target, subtending $0.75^{\circ}$, frontprojected onto a video screen $1.72 \mathrm{~m}$ in front of the subject). The stimulus jumped horizontally within a sector of between $-15^{\circ}$ and $+15^{\circ}$ of centre. The eye tracker was calibrated at the start of the session and between tests.

\section{Adaptation paradigm}

The paradigm included seven consecutive test blocks, each of 49 saccade trials-a baseline block (simple reflexive paradigm, no intra-saccadic target displacements, see Figure 1(a)), followed by five adaptation blocks (with intra-saccadic target displacements, see Figure 1(b) and (c)), followed by a final test block, which was a repetition of the baseline block, as seen in Figure 1(d).

In the two blocks without intra-saccadic target displacements (baseline block and final test block), the stimulus initially appeared at the centre of the screen. It then disappeared and immediately re-appeared at a different location $(14,16,18,20$, 22 or $24^{\circ}$ left or right of the previous position) with a simultaneous tone and remained at that position for a variable length of time $(1000-2000 \mathrm{~ms})$. The subject made a saccade to the new location, which served as the starting point for the next trial.

In the five adaptation blocks, the stimulus was always displaced centripetally by $12.5 \%$ of its initial amplitude when the initial (primary) saccade reached a $30^{\circ} \mathrm{s}^{-1}$ threshold. This displacement was always completed before the eyes completed the saccade to the next position [38]. The displaced target then remained in place for $1000-2000 \mathrm{~ms}$ to allow the subject to re-fixate.
At the beginning of the adaptation process (see Figure $1(b)$ ), the eyes simply followed the stimulus, resulting in a primary saccade $\left(\mathrm{Sacc}_{\text {prim }}\right)$ in response to the initial stimulus movement $\left(\mathrm{Stim}_{\text {init }}\right)$ and a corrective saccade in response to the intra-saccadic target displacement. At the end of the adaptation process (see Figure 1(c)), the stimulus was still being displaced, but the primary saccades were now directed towards the displaced stimulus position and only few centripetally-directed corrective saccades were present. Hence, the saccadic system learned to 'ignore' the initial stimulus movement in favour of direct saccades to the 'expected' final (i.e. displaced) stimulus position. This 'artificial' hypometria remained in the final (post-adaptation) test block (see Figure $1(d)$ ), where the stimulus was no longer displaced. Consequently, the primary saccades were hypometric, falling short of the initial (and in this block also final) stimulus position and were followed by corrective movements, resulting in a 'step-like' pattern of most saccades in this final block.

The key measure was the gain of the primary saccade $\left(G_{\mathrm{p}}=\mathrm{Sacc}_{\mathrm{prim}} / \mathrm{Stim}_{\mathrm{init}}\right)$ made to the initial stimulus position, where $\mathrm{Sacc}_{\text {prim }}$ is the size of the primary saccade towards the stimulus and $\mathrm{Stim}_{\text {init }}$ is the initial stimulus amplitude before displacement.

\section{Data analysis}

Mixed-design ANOVA was used to analyse differences in mean saccade gain between the baseline block and the final (fifth) adaptation block for each group. The between-group variable was Group (CHI vs control). The within-group variable was Adaptation (baseline vs adaptation block 5). For this analysis, adaptation block 5 was used rather than the final baseline block, as the mean primary gain of the final (post-adaptation) block, while still being hypometric, already incorporated a small amount of 'un-learning', that is the readjustment of saccadic gain in response to the missing target displacements in the final test block. Differences between groups were considered statistically significant at a two-tailed $p$ value $\leq 0.05$.

In addition, a comparison of exponential regression curves fitted to the adaptation data set (adaptation block 1-5) of each subject was conducted. Regression curves were fitted based on non-linear estimation using the function

$$
G(n)=G_{i}-\left(G_{i}-G_{o}\right) e^{-n / \tau}
$$

for each saccade trial $n$ over all 245 saccade trials across the five consecutive adaptation blocks, where $G_{\text {o }}$ represents the initial primary gain value at the beginning of the adaptation process and $G_{\mathrm{i}}$ the 
(a)

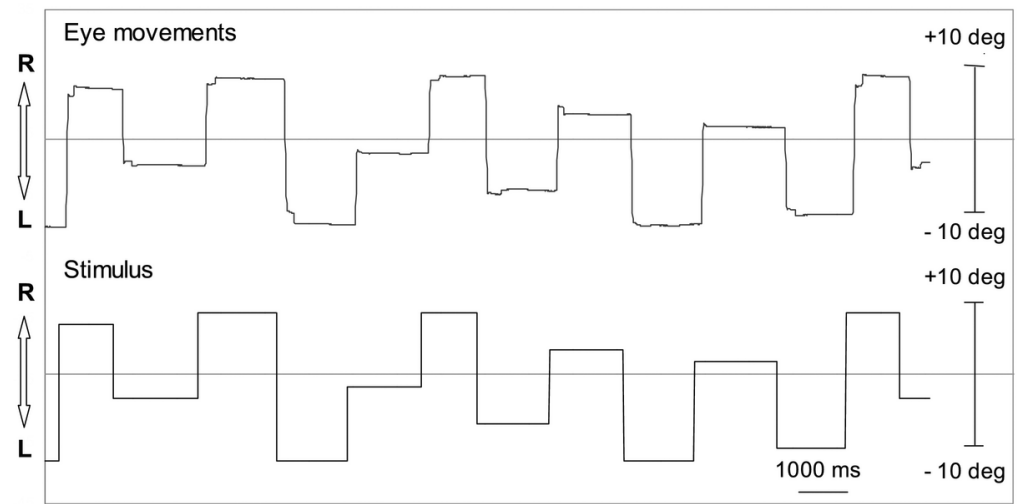

(b)

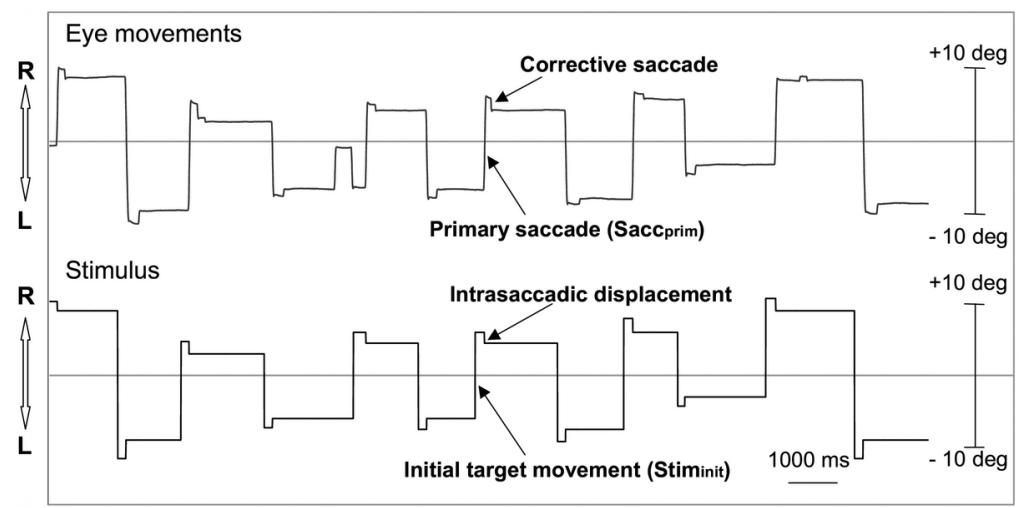

(c)

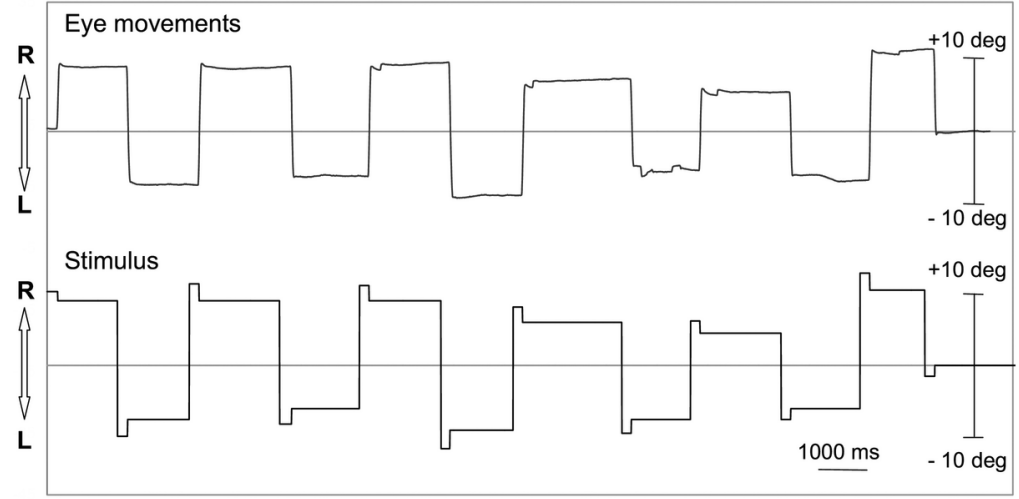

(d)

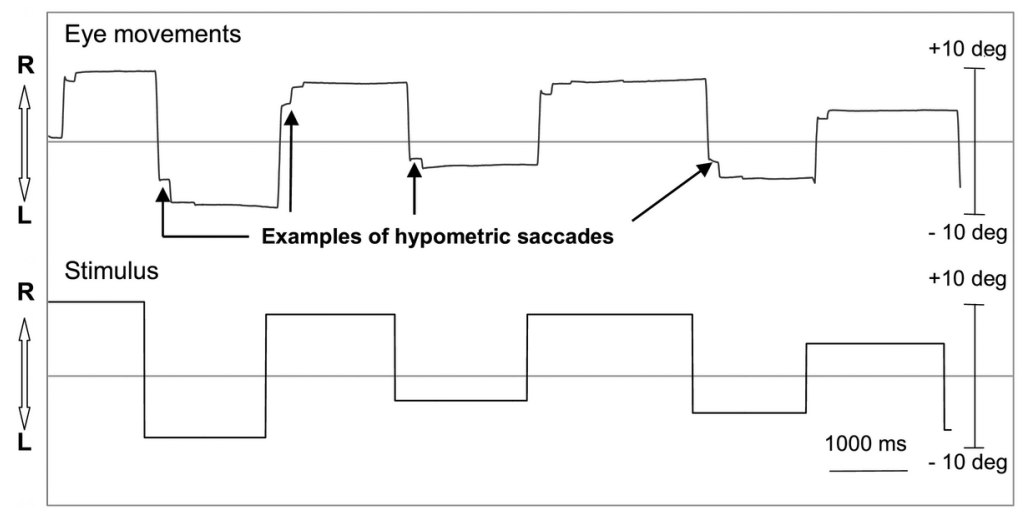

Figure 1. Adaptive paradigm. Line traces represent stimulus position and eye position. Upward line displacements describe stimulus and eye movements to the right, downward to the left. Sample recordings displaying the stimulus and a subject's performance are shown for the initial (pre-adaptation) baseline block (a), no intra-saccadic target displacements, the first of five adaptation blocks $(b)$, with intra-saccadic target displacements, the last adaptation block $(c)$, with intra-saccadic target displacements and the final (post-adaptation) test block $(d)$, no intra-saccadic target displacements. 
asymptotic level of gain. The parameter $\tau$ serves as a measure of the half-life of the time (i.e. number of saccade trials) taken for gain to reach the asymptotic level.

\section{Results}

There was a statistically significant main effect for the within-group variable Adaptation (i.e. before vs after) $(F(1,29)=287.06, p<0.0001)$, showing that both the group with $\mathrm{CHI}$ and the controls adapted in response to the test paradigm. Conversely, there was no statistically significant main effect for the between-group variable Group (i.e. control vs $\mathrm{CHI}$ ) $(F(1,29)=0.75, p=0.39)$, showing that the mean gain of the primary saccades did not differ between the groups. Furthermore, there was no statistically significant interaction effect between Group and Adaptation $(F(1,29)=0.51, p=0.48)$, indicating that there was no significant difference in the extent of adaptation between the subjects with $\mathrm{CHI}$ and the controls (see Figure 2).

Further investigation using exponential regression analysis of the individual adaptation curves (see Figure $3(a)$ ) produced similar findings. In nearly $30 \%$ of subjects in both groups (nine $\mathrm{CHI}$ and eight control subjects), an exponential regression line could not be fitted in a meaningful way (see Figure $3(b)$ ), resulting in a near linear fit with correspondingly distorted $G_{\mathrm{i}}$ and $\tau$ values. Including these cases in the analysis distorted the mean $G_{\mathrm{i}}$-value (ideally between 0.85-0.9) to a large negative figure. Subjects with 'linear fits' were, therefore, removed from the comparison of the key parameters $G_{\mathrm{i}}$ and $\tau$. Subsequent analysis of these variables using a $t$-test for independent samples showed no significant differences in adaptation (CHI vs controls: mean $G_{\mathrm{i}}$ of 0.88 vs 0.86 , $p=0.44$; mean $\tau$ of 100.6 vs $117.6, p=0.76$ ). These results indicate that both the participants with $\mathrm{CHI}$ and the control subjects adapted with equal speed and by an equal amount.

\section{Discussion}

The group with CHI showed no differences in reflexive saccade adaptation and both groups adapted equally well in response to the paradigm. These results indicate that neural damage caused by mild CHI does not impair reflexive saccadic adaptation.

The observation of preserved implicit oculomotor learning after mild $\mathrm{CHI}$ is consistent with earlier reports of preserved implicit cognitive learning, but impaired explicit cognitive learning after head trauma [39-41]. The current subjects were also assessed on several neuropsychological tests with high cognitive loads, including the California Verbal Learning Test (CVLT), a measure of explicit cognitive learning. Similar to other neuropsychological studies after mild head trauma, the group with CHI displayed deficits on the CVLT [14], demonstrating impaired explicit learning processes.

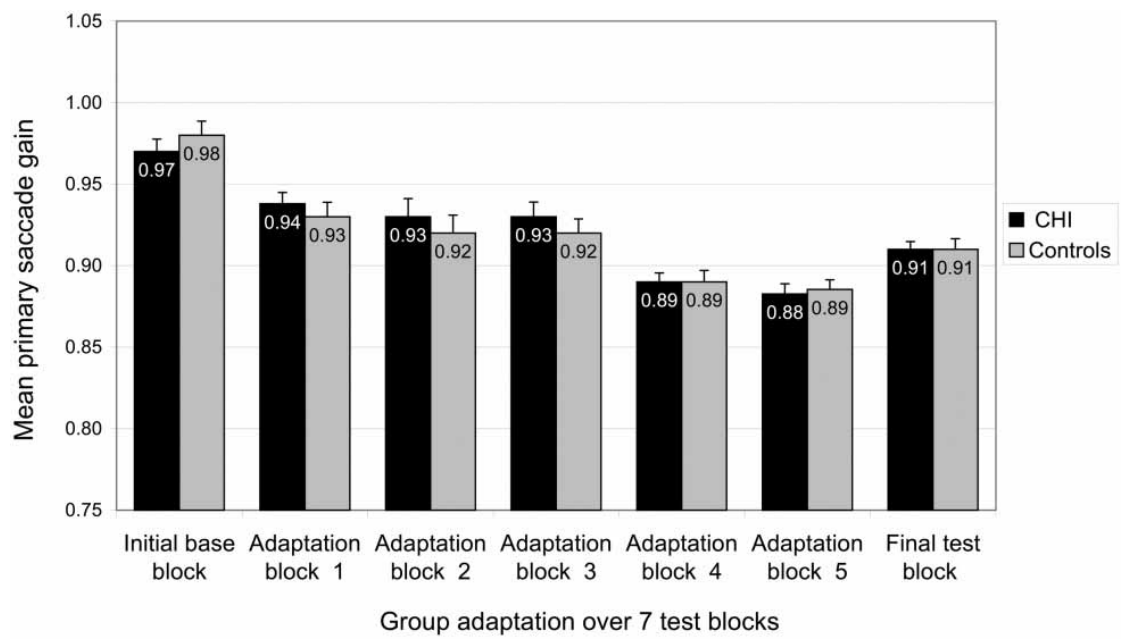

Figure 2. Mean saccadic adaptation over all paradigm blocks in the experiment. In the initial baseline block, both groups showed (normal) slight hypometria in their primary saccades towards the stimulus. Over the course of the adaptation blocks 1-5, both the CHI group and the controls adjusted the gain of their primary saccade in order to direct their gaze towards the final (i.e. centripetally displaced) stimulus position. This (subconscious) adaptation process resulted in increasing hypometria relative to the initial (pre-displacement) stimulus position. This hypometria remained in the final (postadaptation) test block (without target displacements). Note that the mean primary saccade gain in the final test block was larger than in adaptation block 5, showing that the saccadic system had already started to readjust the learned hypometria centrifugally in response to the non-displacing stimulus. 

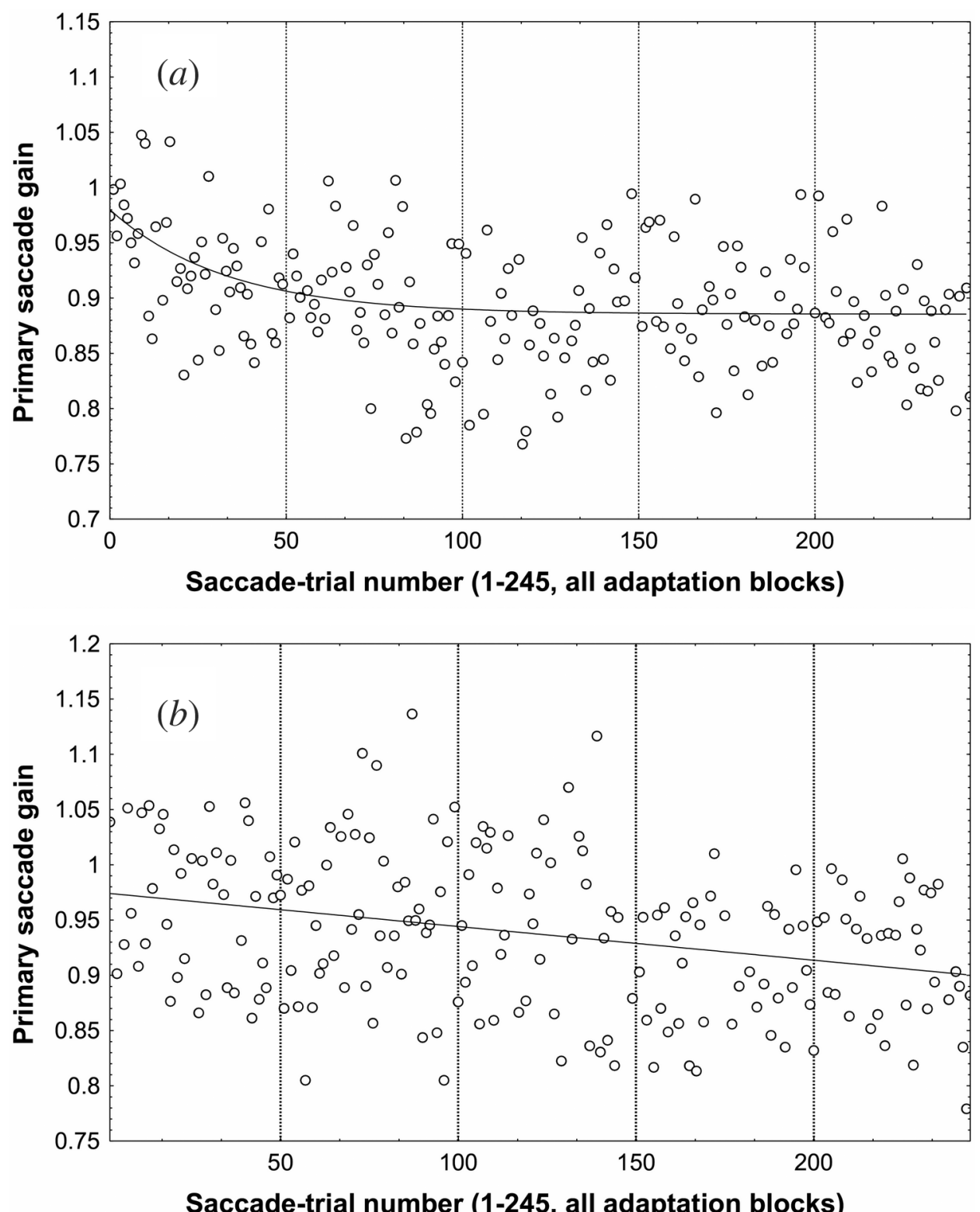

Figure 3. Exponential regression curves fitted to the adaptation blocks $1-5$ of two control subjects ( $a$ and $b$, respectively). Shown are examples of a successful exponential fit $(a)$ and an 'exponential' fit with almost linear characteristics $(b)$.

In nearly a third of subjects in each group, the profile of the saccade adaptation did not seem to occur in the form of an exponential gain decrease but appeared to follow a linear trend. Straube et al. [32] reported a similar phenomenon in an unspecified number of their subjects. While this seems to support the interpretation that the classic exponential learning 'curve' [42] might not be a universal feature of saccadic gain adaptation, the present results may not warrant the conclusion that there are sub-types of 'linear' and 'non-linear' implicit learners. The authors speculate that the observation of 'linear curves' may have been due to large inter-trial variability obscuring the proper fit of an exponential regression line in a sub-set of subjects.
Previous evidence indicates that adaptation of reflexive saccades occurs predominantly in the cerebellum [31-34, 43]. Thus, the present finding of normal saccadic adaptation indicates that cerebellar function is largely spared in mild CHI and supports the interpretation of preserved functional integrity of cerebro-thalamo-cortical communication, which is thought to be essential to saccadic adaptation processes [44]. This interpretation is supported by the report of largely preserved oculomotor smooth pursuit after mild CHI [14], as this oculomotor function is also mediated by, amongst other structures, the cerebellum $[45,46]$.

The deficits of volitional saccades in the same patient group, such as increased saccadic latencies, 
decreased number of self-paced saccades, higher number of directional errors on memory-guided sequences and decreased saccade accuracy of antisaccades and memory-guided saccades $[13,14]$ indicate the disruption of saccadic functions originating in frontal and dorso-parietal cortical areas. Since cortical areas such as the frontal eye field and the dorsolateral pre-frontal cortex are thought to be involved in the adaptation of volitional saccades [35], the authors believe that the adaptation of memoryguided saccades or other volitional saccades might be adversely affected by mild head trauma. Further studies are warranted to examine the metric adaptation of volitional saccades following head trauma.

The current findings indicate that neural injury resulting from mild CHI with a loss of consciousness of limited duration is not likely to reach deeper cerebral structures, such as the thalamus, superior colliculus, basal ganglia, the brainstem or the cerebellum in the majority of patients. This interpretation is consistent with suggestions of a centripetal gradient of impact forces causing neural damage in CHI [9-11], with the impact forces resulting from mild CHI being too weak to reach deeper brain regions in most cases. Interestingly, this patient group showed simultaneous impairment of motor functions originating in frontal and parietal cortical areas while motor functions originating in subcortical areas and the brainstem were preserved, such as saccadic peak velocities [14] and, as shown in the present experiment, adaptation of reflexive saccades. This finding is intriguing as it suggests that the centripetal injury-gradient of concussion $[9,10]$ and the commonly observed structural pathology of mild CHI is reflected in the profile of eye movement dysfunction after mild head trauma. There is considerable evidence that mild TBI adversely affects predominantly frontal, temporal and parietal cerebral areas, while damage to deeper brain areas is progressively smaller and much less frequent $[3,6,7,47-51]$.

\section{Limitations of the current study}

Despite multiple reports that mild head trauma is highly likely to cause neural injury and subsequent functional deficits, the present study has the limitation of not being able to compare the observed level of motor function to functional imaging evidence (e.g. fMRI of SPECT) of neural injury in the current patient group. Hence, while the detailed knowledge of the functional neuroanatomy of eye movements allows one to make inferences on the location and degree of functional impairment in the brain, and the pattern of impaired and preserved motor functions in the current CHI patient group is consistent with the notion of a centripetal gradient of neural injury, specific correlations between degree and location of neural injury and motor function after mild CHI remain to be examined by future studies.

\section{Concluding remarks}

The preservation of implicit learning and subconscious motor processes suggested by the current experiment adds an important element to the model of a close association between the pattern of oculomotor impairment and the commonly observed cerebral pathology after mild head trauma. These results support earlier notions of a very close relationship between motor performance and the biological status of the injured brain [52] and subsequent suggestions for the utility of instrumented motor testing to gauge the functional integrity of the injured brain independently of psychometric status and patient-self-report [14, 52-54].

\section{Acknowledgements}

The authors wish to thank the Canterbury Medical Research Foundation for its financial contribution to this project.

\section{References}

1. Levin HS, Williams DH, Eisenberg HM, et al. Serial MRI and neurobehavioural findings after mild to moderate closed head injury. Journal of Neurology, Neurosurgery and Psychiatry 1992;55:255-62.

2. Mittl RL, Grossman RI, Hiehle JF, et al. Prevalence of MR evidence of diffuse axonal injury in patients with mild head injury and normal head CT findings. American Journal of Neuroradiology 1994;15:1583-9.

3. Hofman PA, Verhey FR, Wilmink JT, et al. Brain lesions in patients visiting a memory clinic with postconcussional sequelae after mild to moderate brain injury. Journal of Neuropsychiatry and Clinical Neurosciences 2002;14:176-84.

4. Lorberboym M, Lampl Y, Gerzon I, et al. Brain SPECT evaluation of amnestic ED patients after mild head trauma. American Journal of Emergency Medicine 2002;20:310-3.

5. Jane JA, Steward O, Gennarelli T. Axonal degeneration induced by experimental noninvasive minor head injury. Journal of Neurosurgery 1985;62:96-100.

6. Kant R, Smith-Seemiller L, Isaac G, et al. Tc-HMPAO SPECT in persistent post-concussion syndrome after mild head injury: Comparison with MRI/CT. Brain Injury 1997;11:115-24.

7. Chen SH, Kareken DA, Fastenau PS, et al. A study of persistent post-concussion symptoms in mild head trauma using positron emission tomography. Journal of Neurology, Neurosurgery and Psychiatry 2003;74:326-32.

8. Wrightson P, Gronwall D. Mild head injury in New Zealand: Incidence of injury and persisting symptoms. New Zealand Medical Journal 1998;111:99-101.

9. Ommaya AK. Head injury mechanisms and the concept of preventive management: A review and critical synthesis. Journal of Neurotrauma 1995;12:527-46. 
10. Ommaya AK, Gennarelli TA. Cerebral concussion and traumatic unconsciousness. Correlation of experimental and clinical observations of blunt head injuries. Brain 1974;97:633-54.

11. Wilson JT. The relationship between neuropsychological function and brain damage detected by neuroimaging after closed head injury. Brain Injury 1990;4:349-63.

12. Servadei P, Vergoni G, Pasini A, et al. Diffuse axonal injury with brainstem localisation: Report of a case in a mild head injured patient. Journal of Neurosurgical Sciences 1994;38:129-30.

13. Heitger MH, Anderson TJ, Jones RD. Saccade sequences as markers for cerebral dysfunction following mild closed head injury. Progress in Brain Research 2002;140:433-48.

14. Heitger $\mathrm{MH}$, Anderson TJ, Jones RD, et al. Eye movement and visuomotor arm movement deficits following mild closed head injury. Brain 2004;127:575-90.

15. MacAskill MR, Anderson TJ, Jones RD. Saccadic adaptation in neurological disorders. Progress in Brain Research 2002;140:417-31.

16. Leigh RJ, Zee DS. The Neurology of Eye Movements. 3rd ed. New York: Oxford University Press; 1999.

17. Abel LA, Schmidt D, Dell'Osso LF, et al. Saccadic system plasticity in humans. Annals of Neurology 1978;4:313-8.

18. Optican LM, Zee DS, Chu FC. Adaptive response to ocular muscle weakness in human pursuit and saccadic eye movements. Journal of Neurophysiology 1985;54:110-22.

19. Cheraskin E. Macular degeneration: How big is the problem? Journal of the National Medical Association 1992;84:873-6.

20. McLaughlin SC. Parametric adjustment in saccadic eye movements. Perception and Psychophysics 1967;2:359-62.

21. Optican LM, Robinson DA. Cerebellar-dependent adaptive control of primate saccadic system. Journal of Neurophysiology 1980;44:1058-76.

22. Optican LM, Zee DS, Miles FA. Floccular lesions abolish adaptive control of post-saccadic ocular drift in primates. Experimental Brain Research 1986;64:596-98.

23. Frens MA, van Opstal AJ. Transfer of short-term adaptation in human saccadic eye movements. Experimental Brain Research 1994;100:293-306.

24. Deubel H. Is saccadic adaptation context-specific? In: Findlay JM, editor. Eye movement research. Amsterdam: Elsevier Science; 1995. pp 177-87.

25. Deubel H. Separate adaptive mechanisms for the control of reactive and volitional saccadic eye movements. Vision Research 1995;35:3529-40.

26. Deubel H, Schneider WX, Bridgeman B. Postsaccadic target blanking prevents saccadic suppression of image displacement. Vision Research 1996;36:985-96.

27. Frens MA, Van Opstal AJ. Monkey superior colliculus activity during short-term saccadic adaptation. Brain Research Bulletin 1997;43:473-83.

28. Takagi M, Zee DS, Tamargo RJ. Effects of lesions of the oculomotor vermis on eye movements in primate: Saccades. Journal of Neurophysiology 1998;80:1911-31.

29. MacAskill MR, Anderson TJ, Jones RD. Adaptive modification of saccade amplitude in Parkinson's disease. Brain 2002;125:1570-82.

30. Bridgeman B, Hendry D, Stark L. Failure to detect displacement of the visual world during saccadic eye movements. Vision Research 1975;15:719-22.

31. Waespe W, Baumgartner R. Enduring dysmetria and impaired gain adaptivity of saccadic eye movements in Wallenberg's lateral medullary syndrome. Brain 1992;115:1123-46.

32. Straube A, Deubel H, Ditterich J, et al. Cerebellar lesions impair rapid saccade amplitude adaptation. Neurology 2001;57:2105-8.
33. Kanayama R, Bronstein AM, Shallo-Hoffmann J, et al. Visually and memory guided saccades in a case of cerebellar saccadic dysmetria. Journal of Neurology, Neurosurgery and Psychiatry 1994;57:1081-4.

34. Desmurget M, Pelisson D, Grethe JS, et al. Functional adaptation of reactive saccades in humans: A PET study. Experimental Brain Research 2000;132:243-59.

35. Deubel H. Separate mechanisms for the adaptive control of reactive, volitional, and memory guided saccadic eye movements. In: Gopher G, Koriat A, editors. Attention and Performance XVII: Cognitive Regulation of Performance. Cambridge, MA: MIT Press; 1999. pp 697-721.

36. Reulen JPH, Marcus JT, Koops D, et al. Precise recording of eye movement: The IRIS technique Part 1. Medical and Biological Engineering and Computing 1988;26:20-6.

37. Muir SR, MacAskill MR, Herron D, et al. EMMA - an eye movement measurement and analysis system. Australasian Physical and Engineering Sciences in Medicine 2003; 26:18-24.

38. MacAskill MR, Muir SR, Anderson TJ. Saccadic suppression and adaptation: Revisiting the methodology. In: Becker W, Deubel H, Mergner H, editors. Current oculomotor research: Physiological and psychological aspects. New York: Plenum; 1999. pp 93-6.

39. Vakil E, Biederman Y, Liran G, et al. Head-injured patients and control group: Implicit versus explicit measures of frequency of occurrence. Journal of Clinical and Experimental Neuropsychology 1994;16:539-46.

40. Shum D, Sweeper S, Murray R. Performance on verbal implicit and explicit memory tasks following traumatic brain injury. Journal of Head Trauma Rehabilitation 1996; 11:43-53.

41. McDowall J, Martin S. Implicit learning in closed head injured subjects: Evidence from an event sequence learning task. New Zealand Journal of Psychology 1996;25:1-6.

42. Straube A, Fuchs AF, Usher S, et al. Characteristics of saccadic gain adaptation in rhesus macaques. Journal of Neurophysiology 1997;77:874-95.

43. Inaba $\mathrm{N}$, Iwamoto $\mathrm{Y}$, Yoshida $\mathrm{K}$. Changes in cerebellar fastigial burst activity related to saccadic gain adaptation in the monkey. Neuroscience Research 2003;46:359-68.

44. Gaymard B, Rivaud-Pechoux S, Yelnik J, et al. Involvement of the cerebellar thalamus in human saccade adaptation. European Journal of Neuroscience 2001;14:554-60.

45. Pierrot-Deseilligny C, Gaymard B. Smooth pursuit disorders. Baillieres Clinical Neurology 1992;1:435-54.

46. Straube A, Scheuerer W, Eggert T. Unilateral cerebellar lesions affect initiation of ipsilateral smooth pursuit eye movements in humans. Annals of Neurology 1997; 42:891-98.

47. Gray BG, Ichise $M$, Chung DG, et al. Technetium99m-HMPAO SPECT in the evaluation of patients with a remote history of traumatic brain injury: A comparison with $\mathrm{X}$-ray computed tomography. Journal of Nuclear Medicine 1992;33:52-8.

48. Abu-Judeh HH, Parker R, Singh $M$, et al. SPET brain perfusion imaging in mild traumatic brain injury without loss of consciousness and normal computed tomography. Nuclear Medicine Communications 1999;20:505-10.

49. Jacobs A, Put E, Ingels $M$, et al. One-year follow-up of technetium-99m-HMPAO SPECT in mild head injury. Journal of Nuclear Medicine 1996;37:1605-9.

50. Otte A, Ettlin TM, Nitzsche EU, et al. PET and SPECT in whiplash syndrome: A new approach to a forgotten brain? Journal of Neurology, Neurosurgery and Psychiatry, 1997;63:368-72.

51. Bicik I, Radanov BP, Schafer N, et al. PET with 18fluorodeoxyglucose and hexamethylpropylene amine oxime 
SPECT in late whiplash syndrome. Neurology 1998; $51: 345-50$.

52. Reitan RM, Wolfson D. The significance of sensory-motor functions as indicators of brain dysfunction in children. Archives of Clinical Neuropsychology 2003;18:11-8.

53. Swaine BR, Sullivan SJ. Relation between clinical and instrumented measures of motor coordination in traumatically brain injured persons. Archives of Physical Medicine \& Rehabilitation 1992;73:55-9.

54. Heitger MH, Anderson TJ, Jones RD. Long-term eye and arm movement deficits following mild closed head injury - use of computerized motor testing to supplement patient assessment (Abstract). Brain Injury 2003; $17: 49-50$. 